\section{Qualidade de vida e esgotamento profissional entre docentes da rede pública de Ensino Médio e Fundamental no Sul do Brasil}

\author{
Quality of life and burnout among public high \\ school and primary school teachers in \\ Southern Brazil
}

\author{
1 Programa de Pós-graduação \\ em Saúde e Comportamento. \\ Universidade Católica de \\ Pelotas, Pelotas, Brasil. \\ 2 Faculdade de Medicina, \\ Universidade Federal de \\ Pelotas, Pelotas, Brasil. \\ ${ }_{3}$ Curso de Psicologia, \\ Universidade Católica de \\ Pelotas, Pelotas, Brasil. \\ Correspondência \\ V. P. Tabeleão \\ Programa de Pós-graduação \\ em Saúde e Comportamento, \\ Universidade Católica de \\ Pelotas. \\ Rua Dom Pedro II 509, \\ apto. 102, Pelotas, RS \\ 96010-300, Brasil. \\ vivianetabeleao@ig.com.br
}

\section{Abstract}

In order to investigate quality of life among public schoolteachers in relation to socio-demographic characteristics and work conditions, a cross-sectional study was conducted in a sample of 601 primary and secondary teachers from the State and Municipal public school system in the urban area of Pelotas, Rio Grande do Sul State, Brazil. The study analyzed the following domains from the WHOQOL-BREF scale: physical health, psychological health, social relationships, and environment. The mean indices were: 69.2 (SD = 16.8) for physical health, $70.6(S D=14.0)$ for $p s y$ chological health, 72.5 (SD = 17.3) for social relationships, and 60.7 (SD = 14.0) for environment. Age, time in the teaching career, and total number of students were not significantly associated with quality of life. Teachers in municipal schools scored higher than their counterparts in the State public schools in the physical health domain ( $p$ =0.026). Men scored higher than women in the physical and psychological health domains. Higher family income was associated with better quality of life. Higher classroom workload was associated with better scores in the physical health and environment domains.

Quality of Life; Occupational Health; Faculty; Working Environmental
Viviane Porto Tabeleão 1

Elaine Tomasi 2

Siduana Facin Neves 3

\section{Introdução}

Em um cenário de grandes transformações tecnológicas e sociais, são estabelecidas novas necessidades nas relações de trabalho, e também surgem grandes e complexos problemas de toda a natureza afetando os trabalhadores.

O trabalho é uma atividade de caráter social, formador de identidade e desenvolvimento pessoal e, portanto, gerador ou não de saúde e qualidade de vida. Entende-se que saúde e qualidade de vida estabelecem entre si complexas relações, dependentes de um contexto sociocultural e econômico, e também de questões físicas e emocionais individuais.

Em 2004, no Brasil, a atividade docente empregava cerca de $2 \%$ da população economicamente ativa 1 , constituindo-se a preocupação com os índices de qualidade de vida e saúde dos docentes uma importante pauta na agenda da gestão do sistema educacional.

O trabalho docente pode ser caracterizado por baixa remuneração, inadequação estrutural das instituições, superlotação nas salas de aula.

Devido aos baixos salários, os docentes estendem sua carga horária em outras atividades, situação que é agravada pela falta de pausas para descanso, o que acaba gerando desconforto e em alguns casos adoecimento. Esse adoecimento pode ser físico e/ou psíquico, contribuindo para os altos níveis de absenteísmo e abandono de emprego. $\mathrm{O}$ ambiente de trabalho e os fatores psi- 
cossociais têm sido considerados os maiores causadores de problemas de saúde em professores ${ }^{2}$.

O trabalho do docente sempre inclui outros sujeitos na mais tenra idade e em plena formação. Quando ocorrem prejuízos nas condições de trabalho e, consequentemente, na qualidade de vida do professor, há um efeito multiplicador na vida dos demais sujeitos envolvidos e no resultado do seu próprio trabalho.

Apesar de não haver um consenso sobre o conceito de qualidade de vida, ela foi definida pelo The WHO-QOL Group como " a percepção do indivíduo sobre a sua posição na vida, no contexto da cultura e dos sistemas de valores nos quais ele vive, e em relação aos seus objetivos, expectativas, padrões e preocupações" 3 (p. 1403).

Para aferir a qualidade de vida, diversos estudos têm utilizado o WHOQOL-bref, que é um instrumento validado pela Organização Mundial da Saúde (OMS), e mede a qualidade de vida em quatro domínios: físico, psicológico, relações sociais e ambiente 4 .

Ainda são escassos na literatura os estudos dedicados ao conhecimento sobre as condições de trabalho e a qualidade de vida de docentes da rede pública de ensino. Este estudo teve como objetivo investigar os níveis de qualidade de vida entre docentes de escolas estaduais e municipais, de ensinos Fundamental e Médio, e relacioná-los com características sociodemográficas e do processo de trabalho.

\section{Métodos}

O estudo teve delineamento transversal e foi realizado em Pelotas, Rio Grande do Sul, Brasil, em 2008. Esse delineamento é adequado para a investigação de eventos com alta prevalência na população de referência, como é o caso do desgaste profissional e da percepção de qualidade de vida. Foi uma estratégia metodológica adequada, eficiente e rápida, dados os objetivos do estudo. O município dispunha de uma rede com 105 escolas de ensinos Fundamental e Médio, entre municipais e estaduais. Foram selecionadas 18 escolas por amostragem estratificada e proporcional ao número de docentes. Após contato e autorização da direção, os questionários foram autoaplicados, sob orientação de auxiliares de pesquisa, especialmente capacitados para este fim. As variáveis dependentes foram os quatro domínios da escala de qualidade de vida WHOQOL-bref 4: físico, psicológico, relações sociais e ambiente.

No domínio físico estão aspectos como dor e desconforto, energia e fadiga, sono e repouso; no domínio psicológico incluem-se sentimentos positivos, pensamento, aprendizagem, memória e concentração, autoestima, imagem corporal, e aparência e sentimentos negativos; o das relações sociais engloba as relações pessoais, suporte (apoio) social e atividade sexual; e o domínio ambiente está relacionado à segurança física e proteção, ambiente no lar, recursos financeiros, disponibilidade e qualidade de cuidados de saúde e sociais, oportunidades de adquirir novas informações e habilidades, oportunidades e participação em atividades de recreação e lazer; ambiente físico (poluição/ruído/trânsito/clima) e transporte.

O instrumento possui 26 questões, é validado para o Brasil e os escores variam de 0 a 100 . Valores mais altos indicam melhor qualidade de vida.

As variáveis independentes foram agrupadas em características sociodemográficas (idade e renda individual) e características do trabalho (tempo de docência total, número de alunos, nível de ensino, horas semanais na escola e atividade fora de sala de aula). Para o burnout foi utilizada a Escala de Maslach 5, composta de três fatores: exaustão, despersonalização e realização pessoal, num total de 22 itens.

$\mathrm{Na}$ análise bivariada, utilizou-se o teste t para comparar as médias dos domínios de qualidade de vida entre categorias de variáveis dicotômicas, e o teste F para comparação entre as categorias de variáveis ordinais e nominais com mais de duas categorias. A variável de estratificação foi o tipo de escola - municipal ou estadual. Para a análise multivariada usou-se a regressão linear múltipla, obedecendo ao modelo hierárquico de determinação, no qual idade, tempo de trabalho e renda foram considerados como potenciais fatores de confusão.

A participação dos docentes ocorreu por meio de consentimento assinado, e a confidencialidade da informação individual e o direito de recusa em participar foram plenamente garantidos. $\mathrm{O}$ projeto de pesquisa foi aprovado pelo Comitê de Ética em Pesquisa da Universidade Católica de Pelotas, pelo processo 07/2008, de acordo com a Resolução $n^{\circ}$. 196/96 do Conselho Nacional de Saúde.

\section{Resultados}

No total, 601 docentes responderam ao questionário nas 18 escolas, tendo-se $17 \%$ de recusas entre 726 convidados. Metade da amostra foi oriunda de escolas municipais, configurando-se um equilíbrio perfeito da amostra entre as redes.

A Tabela 1 descreve as características da amostra total e por tipo de escola. 
Descrição da amostra de docentes da rede pública de acordo com as variáveis demográficas e do processo de trabalho e o tipo de escola. Pelotas, Rio Grande do Sul, Brasil, 2008.

\begin{tabular}{|c|c|c|c|c|c|}
\hline & \multicolumn{2}{|c|}{ Amostra } & \multirow{2}{*}{$\begin{array}{c}\text { Rede municipal } \\
\%\end{array}$} & \multirow{2}{*}{$\begin{array}{c}\text { Rede estadual } \\
\%\end{array}$} & \multirow[t]{2}{*}{ Valor de $p$} \\
\hline & n & $\%$ & & & \\
\hline Sexo $[n=595]$ & & & & & 0,206 \\
\hline Masculino & 92 & 15,5 & 17,5 & 13,4 & \\
\hline Feminino & 503 & 84,5 & 82,5 & 86,6 & \\
\hline Idade (anos) [n = 593] & & & & & $<0,001$ \\
\hline $21-38$ & 187 & 31,5 & 38,1 & 25,1 & \\
\hline $39-46$ & 188 & 31,7 & 34,4 & 29,1 & \\
\hline $47-68$ & 218 & 36,8 & 27,6 & 45,8 & \\
\hline Renda familiar per capita (salários mínimos) [n = 518] & & & & & 0,535 \\
\hline \multicolumn{6}{|l|}{ Até 1,5 } \\
\hline $1,6-3,0$ & 164 & 31,7 & 32,1 & 31,3 & \\
\hline 3,1 e mais & 208 & 40,2 & 41,5 & 39,0 & \\
\hline Tempo de docência (anos) $[\mathrm{n}=591]$ & & & & & $<0,001$ \\
\hline Até 10 & 205 & 34,5 & 41,5 & 28,1 & \\
\hline $10,1-20$ & 195 & 33,0 & 32,0 & 34,2 & \\
\hline Mais de 20 & 191 & 32,3 & 26,5 & 37,7 & \\
\hline Horas semanais na escola [ $n=594]$ & & & & & $<0,001$ \\
\hline Até 20 & 370 & 62,3 & 55,4 & 69,0 & \\
\hline Mais de 20 & 224 & 37,7 & 44,6 & 31,0 & \\
\hline Número total de alunos [ $n=509]$ & & & & & $<0,01$ \\
\hline Até 65 & 128 & 25,1 & 30,9 & 18,5 & \\
\hline $66-150$ & 142 & 27,9 & 24,6 & 31,5 & \\
\hline $151-270$ & 113 & 22,2 & 24,6 & 20,2 & \\
\hline Mais de 270 & 126 & 24,8 & 19,9 & 29,8 & \\
\hline Total & 601 & 100,0 & 49,4 & 50,6 & \\
\hline
\end{tabular}

A maioria dos docentes era do sexo feminino (85\%), distribuindo-se de forma semelhante entre os dois tipos de escolas. A idade dos docentes variou de 21 a 68 anos, com média de 43 anos (desvio-padrão-DP = 9,2 anos), sendo os da rede municipal significativamente mais jovens do que os da rede estadual. Quase metade (45\%) daqueles com mais de 46 anos estava na rede estadual, ao passo que menos de $30 \%$ de professores desta faixa etária estão na rede municipal.

Para todos os professores, a renda familiar média foi de 2,5 salários mínimos ( $\mathrm{DP}=1,7 \mathrm{SM}$ ) e não apresentou diferença significativa entre os tipos de escola, apesar de que, ao considerar somente a renda individual, viu-se que a proporção de docentes com mais de três salários mínimos foi maior nas municipais (59\%) do que nas estaduais (52\%).

Seguindo o mesmo padrão da idade, o tempo de docência foi significativamente maior na rede estadual, pois quase $40 \%$ desses professores trabalhavam há mais de 20 anos, ao passo que $42 \%$ dos que referiram menos de 10 anos de docência estavam na rede municipal.

Pouco mais de um terço dos professores cumpria mais de 20 horas semanais na escola, sendo esta proporção significativamente maior na rede municipal.

Um quarto dos docentes era responsável por mais de 270 alunos, sendo que na rede municipal esta proporção foi de $20 \%$ e na estadual chegou a $30 \%$.

Em relação aos indicadores de desgaste profissional, registrou-se que $63 \%$ e $21 \%$ dos docentes apresentaram, respectivamente, níveis médios e altos de exaustão. Um terço de todos os docentes (30\%) apresentou altos níveis de despersonalização, e 58\% e 14\% apresentaram níveis médios e baixos de realização pessoal, respectivamente. A combinação desses indicadores 
resultou em uma prevalência global de burnout de $31 \%$.

Ao analisar os componentes do desgaste por tipo de escola, viu-se que os da rede estadual estavam com maiores índices de exaustão e despersonalização do que seus colegas da rede municipal, ambos com diferenças estatisticamente significativas. O mesmo não foi observado para a realização pessoal (Figura 1). As diferenças para exaustão e despersonalização permaneceram mesmo após o ajuste para idade e tempo de trabalho, variáveis que apresentaram distribuição desigual entre as escolas.

Para o conjunto da amostra, os índices médios nos domínios da escala de qualidade de vida foram: 69,2 (DP = 16,8) para o domínio físico, 70,6 $(\mathrm{DP}=14,0)$ para o psicológico, 72,5 $(\mathrm{DP}=17,3)$ para o de relações sociais e $60,7(\mathrm{DP}=14,0)$ para o domínio meio ambiente.

A Tabela 2 apresenta as médias nos níveis de qualidade de vida de acordo com as variáveis sociodemográficas e do processo de trabalho.

As variáveis idade, tempo de docência e número total de alunos não se associaram significativamente à pontuação nos domínios da qualidade de vida. Docentes das escolas municipais pontuaram mais no domínio físico do que seus colegas da rede estadual ( $p=0,026$ ), sem diferenças importantes para os demais domínios. Ao ajustar para renda, essa associação desapareceu.
Os homens estavam melhores do que as mulheres somente nos domínios físico e psicológico. Para todos os domínios, quanto maior a renda familiar, mais alta a pontuação na qualidade de vida. Quanto maior a carga horária na escola, melhor a pontuação nos domínios físico e ambiente, mantida após ajuste para renda e tempo de trabalho.

A Tabela 3 apresenta as médias nos níveis de qualidade de vida de acordo com as variáveis do desgaste profissional. Sem exceção, todos os indicadores da síndrome de burnout se associaram significativamente com a qualidade de vida em todos os domínios, evidenciando que quanto maior o desgaste, menor a qualidade de vida.

\section{Discussão}

A proporção de recusas registrada pelo estudo, apesar de expressiva, não foi diferente entre os tipos de escolas, e os principais motivos alegados pelos docentes foi a falta de tempo e o temor de que os resultados pudessem prejudicá-los de alguma forma.

Apesar disso, o perfil demográfico desta amostra foi bastante similar ao da categoria docente de níveis fundamental e médio do Brasil. Os resultados são semelhantes aos achados no Censo do Magistério da Educação Básica no

Figura 1

Ocorrência da síndrome de burnout em docentes da rede pública de acordo com o tipo de escola. Pelotas, Rio Grande do Sul, Brasil, 2008.

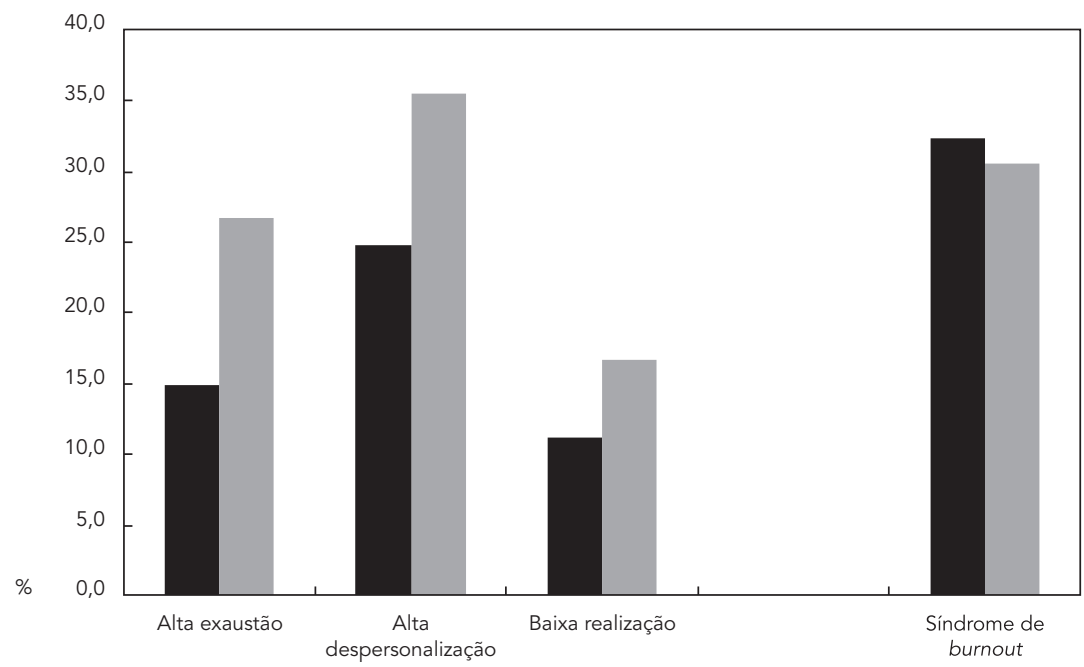


Escores médios nos domínios de qualidade de vida de acordo com o tipo de escola, variáveis demográficas e do processo de trabalho. Pelotas, Rio Grande do Sul, Brasil, 2008

\begin{tabular}{|c|c|c|c|c|}
\hline & \multicolumn{4}{|c|}{ Domínio da escala de qualidade de vida } \\
\hline & \multicolumn{4}{|c|}{ [escore médio (DP)] } \\
\hline & Físico & Psicológico & Relações sociais & Ambiente \\
\hline \multicolumn{5}{|l|}{ Tipo de escola } \\
\hline Estadual & $67,6(17,4)$ & $70,2(14,3)$ & $72,1(17,4)$ & $59,9(14,2)$ \\
\hline Municipal & $70,7(16,1)$ & $71,1(13,7)$ & $73,0(17,2)$ & $61,5(13,8)$ \\
\hline Valor de p & 0,026 & 0,417 & 0,547 & 0,151 \\
\hline \multicolumn{5}{|l|}{ Sexo $[n=595]$} \\
\hline Masculino & $73,8(14,2)$ & $74,4(13,0)$ & $75,2(14,5)$ & $62,2(14,2)$ \\
\hline Feminino & $68,4(17,1)$ & $69,9(14,1)$ & $72,0(17,8)$ & $60,4(14,0)$ \\
\hline Valor de p & 0,005 & 0,005 & 0,113 & 0,247 \\
\hline \multicolumn{5}{|l|}{ Idade (anos) } \\
\hline $21-38$ & $70,3(15,4)$ & $70,6(14,9)$ & $73,1(18,9)$ & $60,7(14,1)$ \\
\hline $39-46$ & $68,8(16,2)$ & $69,5(13,3)$ & $70,7(17,3)$ & $60,3(13,5)$ \\
\hline $47-68$ & $68,2(18,8)$ & $71,3(13,8)$ & $73,8(16,0)$ & $61,0(14,4)$ \\
\hline Valor de p & 0,227 & 0,618 & 0,640 & 0,821 \\
\hline \multicolumn{5}{|c|}{ Renda familiar per capita (salários mínimos) [n = 518] } \\
\hline Até 1,5 & $66,2(17,4)$ & $68,4(13,7)$ & $71,2(17,1)$ & $56,9(14,6)$ \\
\hline $1,6-3,0$ & $67,5(16,9)$ & $69,3(14,9)$ & $70,7(17,4)$ & $59,7(13,0)$ \\
\hline 3,1 e mais & $74,0(14,8)$ & $73,4(12,8)$ & $75,3(16,6)$ & $65,2(11,8)$ \\
\hline Valor de p & $<0,001$ & 0,002 & 0,044 & $<0,001$ \\
\hline \multicolumn{5}{|c|}{ Tempo de docência (anos) } \\
\hline Até 10 & $70,0(15,0)$ & $70,6(13,6)$ & $72,3(17,5)$ & $60,8(14,1)$ \\
\hline $10,1-20$ & $67,8(16,3)$ & $69,3(14,3)$ & $72,1(16,7)$ & $59,3(12,8)$ \\
\hline Mais de 20 & $69,4(19,1)$ & $71,9(14,1)$ & $72,9(17,4)$ & $62,0(14,9)$ \\
\hline Valor de p & 0,695 & 0,404 & 0,751 & 0,422 \\
\hline \multicolumn{5}{|c|}{ Horas semanais na escola } \\
\hline Até 20 & $67,8(17,3)$ & $69,8(14,3)$ & $71,8(17,5)$ & $59,6(14,3)$ \\
\hline Mais de 20 & $71,3(15,7)$ & $71,9(13,3)$ & $73,8(16,9)$ & $62,2(13,4)$ \\
\hline Valor de $\mathrm{p}$ & 0,015 & 0,084 & 0,189 & 0,027 \\
\hline \multicolumn{5}{|c|}{ Número total de alunos } \\
\hline Até 65 & $69,4(18,3)$ & $70,1(14,6)$ & $72,4(18,5)$ & $60,9(14,1)$ \\
\hline $66-150$ & $68,7(16,6)$ & $70,9(13,5)$ & $72,4(16,5)$ & $60,3(14,3)$ \\
\hline $151-270$ & $71,3(16,3)$ & $70,6(14,3)$ & $72,8(18,1)$ & $61,0(13,0)$ \\
\hline Mais de 270 & $68,5(17,3)$ & $70,5(14,1)$ & $73,4(16,3)$ & $60,2(14,5)$ \\
\hline Valor de p & 0,977 & 0,860 & 0,636 & 0,805 \\
\hline Total & $69,2(16,2)$ & $70,6(13,9)$ & $72,5(17,3)$ & $60,7(13,9)$ \\
\hline
\end{tabular}

Brasil (2003) 6 , no qual a maioria dos profissionais era do sexo feminino (85\%), de cor branca e a idade variava de 18 a pouco mais de 65 anos, com a média de idade da maioria dos professores entre 35 a 44 anos. O predomínio do sexo feminino parece tratar-se de uma questão histórica, pois esta profissão era uma das poucas possíveis de ser exercida por mulheres.
Porto et al. 7 encontraram predomínio de mulheres (93\%) e Jardim et al. ${ }^{\text {, estudando docentes }}$ da rede pública e do fundamental, encontraram idade média de 42 anos, o que está de acordo com a nossa amostra.

Análises realizadas por Schwartzman ${ }^{9}$ apontaram um melhor nível salarial para a rede estadual do que para a municipal, embora a média 
Escores médios nos domínios de qualidade de vida de acordo com os componentes da síndrome de burnout. Pelotas, Rio Grande do Sul, Brasil, 2008.

\begin{tabular}{|c|c|c|c|c|}
\hline & \multicolumn{4}{|c|}{$\begin{array}{c}\text { Domínio da escala de qualidade de vida } \\
\text { [escore médio (DP)] }\end{array}$} \\
\hline & Físico & Psicológico & Relações Sociais & Ambiente \\
\hline \multicolumn{5}{|c|}{ Exaustão emocional } \\
\hline Baixa & $82,8(11,7)$ & $81,3(9,7)$ & $82,5(15,0)$ & $72,2(11,1)$ \\
\hline Média & $71,1(14,2)$ & $72,7(11,0)$ & $73,1(16,0)$ & $61,7(12,0)$ \\
\hline Alta & $52,7(15,0)$ & $55,6(13,5)$ & $63,5(18,2)$ & $48,1(12,3)$ \\
\hline Valor de p & $<0,001$ & $<0,001$ & $<0,001$ & $<0,001$ \\
\hline \multicolumn{5}{|c|}{ Despersonalização } \\
\hline Média & $71,9(15,8)$ & $73,7(12,4)$ & $75,17(16,6)$ & $63,3(13,3)$ \\
\hline Alta & $62,4(17,1)$ & $63,6(14,7)$ & $66,4(17,4)$ & $54,5(13,8)$ \\
\hline Valor de $p$ & $<0,001$ & $<0,001$ & $<0,001$ & $<0,001$ \\
\hline \multicolumn{5}{|c|}{ Realização pessoal } \\
\hline Baixa & $57,3(16,8)$ & $59,0(16,7)$ & $63,0(17,4)$ & $53,2(14,2)$ \\
\hline Média & $68,4(15,9)$ & $69,5(12,8)$ & $71,4(17,1)$ & $59,4(13,7)$ \\
\hline Alta & $76,6(14,9)$ & $78,5(9,6)$ & $79,2(15,0)$ & $66,5(11,9)$ \\
\hline Valo de $\mathrm{p}$ & $<0,001$ & $<0,001$ & $<0,001$ & $<0,001$ \\
\hline Total & $69,2(16,2)$ & $70,6(13,9)$ & $72,5(17,3)$ & $60,7(13,9)$ \\
\hline
\end{tabular}

salarial tenha sido praticamente a mesma da encontrada em Pelotas. Em Belo Horizonte (Minas Gerais), a renda pessoal da maioria dos docentes em 2004 era de 4,5 salários mínimos, segundo

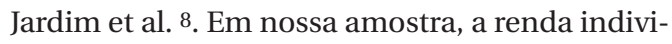
dual foi de 3,5 salários mínimos, sem diferenças entre os tipos de escola, demonstrando a homogeneidade da categoria.

Todos os indicadores de qualidade de vida foram levemente superiores aos encontrados em Natal (Rio Grande do Norte) 2. Destaca-se que tanto em Natal quanto em Pelotas o domínio ambiente teve a pior pontuação. $\mathrm{O}$ mesmo resultado foi encontrado entre 237 cirurgiões-dentistas de Goiânia (Goiás) 10 e entre 110 anestesiologistas de Recife (Pernambuco) 11, implicando uma consistência entre as diferentes amostras, o que reforça a adequação do instrumento.

Uma possível explicação para esse achado seria que no domínio ambiente estão incluídos aspectos financeiros e sociais como saúde, educação e lazer.

Investigações realizadas por Codo 12 sobre a saúde mental dos professores de Ensinos Médio e Fundamental em todo o país, abrangendo 1.440 escolas e mais de 50 mil professores, revelaram que $48 \%$ apresentavam algum sintoma da síndrome de burnout e $26 \%$ apresentavam exaustão emocional, com variação de 17\% em Minas
Gerais e Ceará a 39\% no Rio Grande do Sul. Esse resultado foi similar ao encontrado em Pelotas, com $31 \%$ dos docentes classificados com a síndrome. Em Hong Kong (China), Chan 13 registrou um terço dos docentes com sintomas de estresse e burnout. Pesquisa feita com mais de 8 mil professores da educação básica da rede pública na Região Centro-oeste do Brasil revelou que $16 \%$ dos entrevistados apresentavam essa síndrome.

Quanto mais carga de trabalho, melhores os resultados em nossa amostra para os domínios físico e ambiente, resultados diferentes dos de Martinez et al. 14. O mesmo também foi encontrado em um estudo com anestesiologistas 11, indicando uma tendência que se aplica a outras categorias profissionais. O que pode explicar essa aparente contradição é o efeito da renda, pois quem trabalha mais ganha mais e isto sem dúvida contribui para a melhor qualidade de vida, principalmente no domínio ambiente. Em nosso estudo, o ajuste por renda foi importante apenas para o domínio físico, ou seja, não foi observado este comportamento para o domínio ambiente, como encontrado em outras pesquisas.

Em relação ao gênero, nossos resultados apontaram para uma pior qualidade de vida entre as mulheres, o que também foi observado nos estudos de Calumbi et al. 11 e de Martinez et al. 14 . Esses mesmos trabalhos não encontraram asso- 
ciação entre os níveis de qualidade de vida e a faixa etária, a exemplo dos achados de Pelotas.

No Rio Grande do Sul, a rede estadual realizou, no início do ano letivo de 2008, uma modificação no quadro de pessoal das escolas. Em algumas, houve diminuição do número de docentes e substituição dos que estavam em funções administrativas por servidores não-docentes. Foram eliminadas as atividades no turno da noite de alguns estabelecimentos, com redistribuição de seus professores conforme a demanda. Implantou-se o que se chamou de "enturmação", ou seja, reunir alunos de turmas pequenas para formar turmas maiores. Essas modificações podem ter colaborado para a observação de maiores níveis de exaustão e de despersonalização na rede estadual do que na municipal, mantidos após o ajuste para idade e tempo de trabalho.

Acredita-se que os instrumentos utilizados configuram-se como uma aproximação quantitativa aos conceitos mais amplos, tanto de qualidade de vida quanto de desgaste profissional. Os autores que os desenvolveram preocuparamse em oferecer aos pesquisadores que utilizam abordagens quantitativas um meio de aferir tais constructos de forma mais objetiva. É certo que essa aproximação apresenta limitações, mas em virtude dos nossos objetivos, delineamento e amostra, essa foi a alternativa adotada.
Atividades que estimulem a aproximação entre professores podem contribuir para evitar a tendência a expectativas profissionais inalcançáveis, substituindo-as por metas realistas e discutidas coletivamente. A busca por melhores condições de trabalho deve ser contínua frente às entidades de classe e às instituições de ensino.

Seriam úteis estudos voltados à compreensão da adequação entre as mudanças educacionais propostas e implementadas e a realidade que os trabalhadores enfrentam nas escolas. As contradições existentes podem estar na origem da exposição aos fatores de risco para o adoecimento da categoria dos trabalhadores do ensino. Estudos transversais, como o presente, revestem-se de limitações inerentes ao método, assim como a abordagem quantitativa pode revelar-se insuficiente, por si só, para o entendimento das complexas relações que permeiam o trabalho docente e o processo de adoecimento de seus profissionais.

Ainda assim, acredita-se que os resultados serão úteis para refletir e apontar caminhos que levem a ações de prevenção, manutenção e promoção da qualidade de vida no trabalho, podendo traduzir-se em subsídios para os próprios docentes, os gestores e os formuladores de políticas públicas de recursos humanos na área da educação.

\section{Resumo}

Para investigar os niveis de qualidade de vida entre docentes de escolas estaduais e municipais e relacionálos com características sociodemográficas e do processo de trabalho, realizou-se um estudo transversal com 601 docentes da rede pública de Ensino Fundamental e Médio da zona urbana de Pelotas, Rio Grande do Sul, Brasil. Os domínios da escala de qualidade de vida WHOQOL-bref - físico, psicológico, relações sociais e ambiente - foram analisados. Os índices médios foram: 69,2 (desvio-padrão-DP = 16,8) para o domínio físico, 70,6 (DP = 14,0) para o psicológico, 72,5 $(D P=17,3)$ para o domínio relações sociais e 60,7 $(D P=14,0)$ para o ambiente. Idade, tempo de docência e total de alunos não se associaram significativamente à qualidade de vida. Docentes das escolas municipais pontuaram mais no domínio físico do que os da rede estadual $(p=0,026)$. Os homens estavam melhores do que as mulheres somente nos domínios físico e psicológico. Quanto maior a renda familiar, mais alta a pontuação na qualidade de vida. Quanto maior a carga horária na escola, melhor a pontuação nos domínios físico e ambiente.

Qualidade de Vida; Saúde do Trabalhador; Docentes; Ambiente de Trabalho. 


\section{Colaboradores}

V. P. Tabeleão participou da concepção do estudo, da coleta e análise de dados e aprovação da versão final do artigo. E. Tomasi participou da concepção e delineamento do estudo, da análise e interpretação dos dados e da aprovação da versão final do artigo. S. F. Neves participou da concepção do estudo, da interpretação dos dados, da revisão crítica do conteúdo e da aprovação da versão final do artigo.

\section{Referências}

1. Cerqueira AC. Evolução do emprego e dos rendimentos no setor público brasileiro: dados para 1992 e 2002. In: Teixeira M, organizador. Negociação e contratação coletiva da qualificação socioprofissional nas relações capital-trabalho. São Paulo: Escola Sindical de São Paulo; 2005.

2. Fernandes MH, Rocha VM. Impact of the psychosocial aspects of work on the quality of life of teachers. Rev Bras Psiquiatr 2009; 31:15-20.

3. The World Health Organization quality of life assessment (WHOQOL): position paper from the World Health Organization. Soc Sci Med 1995; 41:1403-10.

4. Fleck M, Louzada S, Xavier M. Aplicação da versão em português do instrumento abreviado de avaliação da qualidade de vida "WHOQOL-bref". Rev Saúde Pública 2000; 34:178-83.

5. Malasch C. Entendendo o burnout. In: Rossi A, organizador. Stress e qualidade de vida no trabalho: perspectivas atuais da saúde ocupacional. São Paulo: Editora Atlas; 2005.

6. Instituto Nacional de Estudos e Pesquisas Educacionais Anísio Teixeira. Sinopse do censo dos profissionais do magistério da educação básica: 2003. Brasília: Instituto Nacional de Estudos e Pesquisas Educacionais Anísio Teixeira; 2006.

7. Porto LA, Carvalho FM, Oliveira NF, Silvany AM, Araújo TM, Reis EJF, et al. Associação entre distúrbios psíquicos e aspectos psicossociais do trabalho de professores. Rev Saúde Pública 2006; 40:818-26.
8. Jardim R, Barreto SM, Assunção AA. Condições de trabalho, qualidade de vida e disfonia entre docentes. Cad Saúde Pública 2007; 23:2439-61.

9. Scwartzman S. O crescimento do ensino superior e as mudanças nos salários dos professores. http://www.schwartzman.org.br/sitesimon $/$ ? $\mathrm{p}=172 \&$ lang=pt-br (acessado em 20/Set/2007).

10. Nunes FN, Freire MCM. Qualidade de vida de cirurgiões-dentistas que atuam em serviço público. Rev Saúde Pública 2006; 40:1019-26.

11. Calumbi RA, Amorim JA, Maciel CMC, Damázio O, Teles AJF. Avaliação da qualidade de vida de anestesiologistas da Cidade de Recife. Rev Bras Anestesiol 2010; 60:42-51.

12. Codo W. Educação: carinho e trabalho. 3a Ed. Petrópolis: Editora Vozes; 2002.

13. Chan DW. Hardiness and its role in the stressburnout relationship among prospective Chinese teachers in Hong Kong. Teaching \& Teacher Education 2003; 19:381-95.

14. Martinez KASC, Vitta A, Lopes ES. Avaliação da qualidade de vida dos professores universitários da Cidade de Bauru-SP. Salusvita 2009; 28:217-24.

Recebido em 15/Abr/2011

Versão final reapresentada em 21/Jul/2011

Aprovado em 22/Ago/2011 